\title{
HIGHLIGHTS
}

CANCER

\section{Oncofetal fibronectin mRNA and papillary thyroid cancer}

Quantification of oncofetal fibronectin (onfFN) mRNA may provide a useful supplemental tool to detect circulating thyroid cancer cells in patients who have been treated for papillary thyroid cancer (PTC), particularly while they are on levothyroxine treatment, suggest a team from Hannover Medical School, Germany.

\section{Early detection of recurrent disease in patients ... is vital to optimize prognosis 77}

Early detection of recurrent disease in patients treated for PTC is vital to optimize prognosis. Measurement of serum thyroglobulin, a specific marker of disease recurrence, is part of the standard of care in the follow-up of patients with the disease, but the TSH stimulation used to enhance the test's sensitivity may promote disease recurrence. A drive to develop alternative detection approaches, therefore, exists. "Previously, we demonstrated the usefulness of onfFN transcripts in detecting circulating thyroid carcinoma cells and established a sensitive assay to reliably quantify onfFN mRNA in the peripheral blood," explains Eric Hesse, one of the senior researchers. Now, the team use this assay in 95 patients followed up after treatment for PTC to discover how onfFN mRNA levels correlate with disease status and TSH level.

The investigators used conventional methods, including thyroglobulin measurement, to determine the patients' disease status and also quantified patients' levels of onfFN mRNA in mononuclear cells isolated from peripheral blood both during levothyroxine treatment and after levothyroxine withdrawal. Blood samples from 25 healthy individuals were analyzed to determine a diagnostic cut-off level of onfFN mRNA.

onfFN transcripts were highly abundant in the peripheral blood of the patients, but levels of onfFN mRNA did not differ significantly between patients who were free of disease, had local residual disease or metastatic disease. However, there was a trend towards higher expression rates of onfFN mRNA in patients with either local or distant metastases than those free of disease. Interestingly, during TSH stimulation, a markedly increased fraction of patients in all three disease stages had positive onfFN mRNA tests; discrimination between disease states was better without TSH stimulation.

"We now plan to analyze more patients with differentiated thyroid cancer at different disease stages and also investigate how TSH affects onfFN expression and/or the abundance of circulating tumor cells," concludes Hesse.

Carol Wilson

Original article Wehmeier, M. et al. Oncofetal fibronectin mRNA Is highly abundant in the blood of patients with papillary thyroid carcinoma and correlates with high-serum thyroid-stimulating hormone levels. Thyroid 20, 607-613 (2010) 